\title{
LIMITS OF PLASMA FOCUSING OF HIGH CURRENT ELECTRON BEAMS
}

\author{
S. Adamenko, E. Bulyak *, V. Stratienko, N. Tolmachev Ukraine
}

\begin{abstract}
Limits of the electron beam density in the focus are evaluated. As it shown, the maximal beam density is achievable in the relativistic vacuum diode with the needle anode. In such a device, the initial emittance of a beam is rather small. The electron beam gains emittance while being focused with positive ions into the anode due to collisions with the ions. Based on the paraxial beam model, the beam density in the focus is estimated. As is shown, the lowest limit of focus length is proportional to square root from ratio of the beam particle energy to the initial beam density. The maximal coefficient of the beam initial density multiplication in the focus is proportional to the initial transverse dimension of the beam and square root from the beam current.
\end{abstract}

\section{INTRODUCTION}

The report is aimed at estimation of the limiting parameters for the beams undergo plasma focusing on the needle-like anode.

We study the beam dynamics in the inter-electrode gap where there is no acceleration voltage. Therefore, the beam particle motion is considered as uniform, without acceleration.

\section{MODEL SET-UP}

Let us consider the laminar cylindrical beam of electrons with relativistic factor $\gamma$ and longitudinal density $N_{b}$ entering a system filled with plasma of the density sufficient to neutralize the beam space charge. The beam is focused with the space charge of plasma ions. For the ideal case, the focus spot is of the zero size. Accounting for the pair electron-ion collisions leads to heating of the beam. As a result, the beam phase space is increased yielding increasing the spot size in the focus. Our goal is to estimate the maximal beam density which can be reached in the system under consideration.

Kinetics of the axially symmetrical beam with the transverse distributing function $f\left(r, p_{r} ; p_{\varphi}, t\right)$ can be described as

$$
\frac{\partial f}{\partial t}+\frac{p_{r}}{m} \frac{\partial f}{\partial r}+\frac{p_{\phi}}{m r^{2}} \frac{\partial f}{\partial \phi}+\left(\frac{p_{\phi}^{2}}{m r^{3}}-\frac{\partial U}{\partial r}\right) \frac{\partial f}{\partial r}=0 .
$$

\footnotetext{
${ }^{*}$ Email: bulyak@kipt.kharkov.ua
}

Here $U$ is a potential for the radial force acting on the beam electrons; $p_{\varphi}=\dot{\varphi} \gamma r^{2}$ is the canonical momentum conjugated to the coordinate $\varphi$.

\subsection{Focusing of a laminar collisionless beam}

Let $p_{\varphi}=0$ in (1) and consider the trajectory of a particle at the beam bound.

Potential for the radial force of the completely neutralized beam with the initial radius $a$ based on the fact that the repulsive force $\sim(1-\beta)$ whereas the attractive $\sim-1$, is [1]:

$$
U(r)=-\frac{e^{2} N_{b} \beta^{2}}{2 \pi \epsilon_{0}} \log \frac{r}{a}=-\frac{2 \nu m c^{2}\left(\gamma^{2}-1\right)}{\gamma^{2}} \log \frac{r}{a},
$$

where $\nu \equiv N_{b} r_{0}$ is the Budker parameter equal to production of the longitudinal density $N_{b}$ by the electron classical radius $r_{0}$. It is worth to emphasize that at $\nu=\gamma$ the beam current is equal to the Alfven limit current.

As is seen from (2), the transverse potential is independent on time, hence the corresponding hamiltonian is an invariant of motion:

$$
H=\frac{p_{r}^{2}}{2 \gamma m}+U(r)=\text { const } .
$$

Here we take the transverse motion being nonrelativistic, and the longitudinal velocity being constant. After the simple derivations we arrive at the implicit expression for the beam envelope:

$$
s=\frac{1}{2} \sqrt{\frac{\gamma}{n_{b} r_{0}}} \operatorname{Erf}\left[\sqrt{-2 \log \frac{r}{a}}\right] .
$$

Here $s$ is the longitudinal coordinate (along the $z$-axis); $n_{b}$ is the initial beam density (at the system front end); Erf is the error function. The focus length can be derived from (5):

$$
S_{f}=\frac{a}{2} \sqrt{\frac{\pi \gamma}{\nu}}=\frac{1}{2} \sqrt{\frac{\gamma}{r_{0} n_{b}}},
$$

We should emphasize the fundamental feature of the expression for the shortest possible focus length — this length is proportional to the initial beam radius and square root from the ration of the particle energy to the Budker parameter of the beam. 


\section{BEAM HEATING BY THE SHORT-RANGE COLLISIONS}

As is yielded from the previous section, the fully neutralized beam shrinks into a point (its density is risen to infinity).

Effect, preventing the beam density reach infinity at full neutralization, is the multiple scattering of electrons on the focusing ions. As a result, the initially cold beam possesses definite transverse temperature that yields a finite beam density in the focus spot.

As the radial velocity of particles near the spot is much exceeded that due to the radial temperature, we neglect this impartment and limit our consideration just to the tangential (spinning) partial temperature. This temperature causes the centrifugal repulsive force, $F_{r}=\gamma m v^{2} / r$ acting against the focusing force.

For a collision with an ion, electron's squared transverse momentum is increased by:

$$
\left\langle\Delta p_{i}^{2}+\Delta p_{j}^{2}\right\rangle=\frac{\left.8\left(r_{0} m_{0} c\right)^{2}\right)}{a^{2}} \ln \frac{a}{r_{m}}=\frac{\left.8\left(r_{0} m_{0} c\right)^{2}\right)}{a^{2}} \Lambda .
$$

The speed of the momentum growth is

$$
\dot{\Delta p^{2}}=N_{b} \beta c\left\langle\Delta p^{2}\right\rangle=\frac{8 \nu r_{0}\left(m c^{2}\right)^{2} \beta \Lambda}{a^{2} c} .
$$

As the both transverse degrees of freedom are independent each other, then

$$
\dot{\Delta p_{\phi}^{2}}=\frac{1}{2} \dot{\Delta p^{2}}
$$

Dependence of the tangential momentum on time is

$$
p_{\phi}^{2}(t)=\frac{4 \nu r_{0}\left(m c^{2}\right)^{2} \beta}{c} \int_{0}^{t} \frac{\Lambda(a) d t^{\prime}}{a^{2}\left(t^{\prime}\right)} .
$$

Equation for the RMS beam envelope expressed in dimensionless variables could be written as:

$$
\frac{\mathrm{d}^{2} \rho}{\mathrm{d} \zeta^{2}}+\frac{2 \nu}{\rho \gamma}\left[1-\frac{2 r_{0} \Lambda \gamma}{\left(\gamma^{2}-1\right) r_{a}} \int_{0}^{\zeta} \frac{\mathrm{d} \zeta}{\rho^{2}(\zeta)}\right]=0,
$$

where the space variables: the beam radius $\rho$ and longitudinal coordinate $\zeta$ are related to the initial beam radius $r_{a}$ :

$$
\rho \equiv \frac{r}{r_{a}} ; \quad \zeta \equiv \frac{z}{r_{a}} .
$$

Analysis of the integro-differential equation (9) is rather complicated, nevertheless a few general results are obtainable. As far as the equation contains the only longitudinal beam density $\nu$, energy of particles $\gamma$, and initial beam radius $r_{a}$ as parameters, all the features of focusing are only dependent on them. Then, a term at the integral in (9) comprises a small parameter - ratio of the classical radius of electron $r_{0}$ to the initial beam radius $r_{a}\left(r_{0} / r_{a} \ll 1\right)$ which manifests smallness of the repulsive force.
Estimation for the maximal beam density could be derived under assumption that the limit of focusing is reached if the focusing force is neutralized with the centrifugal one:

$$
1=\frac{2 r_{0} \Lambda \gamma}{\left(\gamma^{2}-1\right) r_{a}} \int_{0}^{\zeta} \frac{\mathrm{d} \zeta^{\prime}}{\rho^{2}\left(\zeta^{\prime}\right)}
$$

Putting roughly $\rho(\zeta) \approx \rho_{m}$, we get the estimated minimal squared beam radius (the reversed magnitude, $\rho^{-2}$ produces the multiplication factor for the initial beam density):

$$
\rho_{m}^{2}=\frac{r_{0} \Lambda \gamma}{\left(\gamma^{2}-1\right) r_{a}} \sqrt{\frac{\pi \gamma}{\nu}}
$$

As it is yielded, the obtained estimated beam radius is in good excess of the classical electron radius.

\section{RESULTS AND SUMMARY}

Estimation of the limit focusing for the initially parallel probe beam with current $I=10 \mathrm{kA}$, energy $E=1 \mathrm{MeV}$ $(\gamma \approx 3)$, and initial radius $r_{a}=50 \mathrm{mkm}$ yields: the focusing length is $S_{f}=95 \mathrm{mkm}$

the maximal possible beam density is

$$
j_{m}=\frac{I^{3 / 2}\left(\gamma^{2}-1\right)^{1 / 4}}{r_{a}} 1.36 \times 10^{11}=3.57 \times 10^{21} \mathrm{~A} / \mathrm{m}^{2}
$$

(the minimal radius is $8.4 \times 10^{-15} \mathrm{~m} \approx 30 r_{0}$ ).

Thus, the results of study the idealized model are physically reasonable. The esteems have to be considered as the ideal, ever achievable parameters. Any deviation from the ideal conditions, such as under neutralization of the beam space charge, or extra density of ions (then capturing electrons), etc., gives rise to the focus spot area.

\section{REFERENCES}

[1] E. Bulyak, Storage of Ions by the Intense Pulsed Electron Beams. Ph.D. Thesis, Kharkov, 1988

[2] S. Heifets, Y.T. Yan, Quantum Effects in Tracking, SLACPUB-773, 1998 